\title{
Aggiornamento sulla nutrizione iodica in Italia: i risultati della seconda sorveglianza dell'Osservatorio per il Monitoraggio della lodoprofilassi in Italia-OSNAMI (2015-2019)
}

\author{
Simona De Angelis ${ }^{1}$. Daniela Rotondi ${ }^{1}$. Enzo Gilardi ${ }^{1}$. Paolo Stacchini ${ }^{2}$. Augusto Alberto Pastorelli ${ }^{2}$. \\ Angela Sorbo ${ }^{2}$. Marilena D'Amato ${ }^{2}$. Anna Chiara Turco ${ }^{2}$. Emanuela Medda ${ }^{3}$. Roberto Da Cas ${ }^{4}$. Sebastiano Andò ${ }^{5}$. \\ Daniela Bonofiglio ${ }^{5}$. Marcello Bagnasco ${ }^{6}$. Maurizio Gasperi ${ }^{7}$. Domenico Meringolo ${ }^{8}$. Caterina Mian ${ }^{9}$. \\ Efisio Puxeddu ${ }^{10}$. Concetto Regalbuto ${ }^{11}$. Mariacarla Moleti ${ }^{12}$. Augusto Taccaliti ${ }^{13}$. Salvatore Ulisse ${ }^{14}$. \\ Massimo Tonacchera ${ }^{15}$. Maria Laura Tanda ${ }^{16}$. Francesco Boi ${ }^{17}$. Valeria Ruggiero ${ }^{17}$. Stefano Mariotti ${ }^{17}$. \\ Carlo Corbetta ${ }^{18} \cdot$ Renzo Ciatti ${ }^{19}$. Elisabetta Tarsi ${ }^{19} \cdot$ Vera Stoppioni $^{19} \cdot$ Nicola Perrotti $^{20}$. Onorina Marasco ${ }^{21}$. \\ Giovanna Scozzafava ${ }^{21}$. Marta Camilot ${ }^{22}$. Francesca Teofoli ${ }^{22}$. Francesca Righetti ${ }^{23}$. Antonio Dimida ${ }^{15}$. \\ Giuseppe Plutino $^{24}$. Elena Carrano ${ }^{24} \cdot$ Roberto Copparoni $^{24}$. Marco Gabbianelli ${ }^{1}$. Paolo Vitti ${ }^{15}$. Antonella Olivieri ${ }^{1}$
}

Accettato: 15 settembre 2021 / Pubblicato online: 26 gennaio 2022

(c) The Author(s), under exclusive licence to Springer Nature Switzerland AG 2022

\section{Introduzione}

Il programma di iodoprofilassi su base volontaria è stato introdotto in Italia nel 2005 con 1'approvazione della Legge n. 55 "Disposizioni finalizzate alla prevenzione del gozzo endemico e di altre patologie da carenza iodica", che definisce le modalità di utilizzo e di vendita del sale iodato destinato al consumo diretto oppure impiegato nella preparazione e conservazione dei prodotti alimentari. La legge prevede la disponibilità obbligatoria del sale iodato in tutti i punti vendita e la possibilità del suo utilizzo nella ristorazione collettiva e nell'industria alimentare. Al fine di verificare l'efficienza e l'efficacia del programma di iodoprofilassi e al fine di fornire informazioni utili per eventuali azioni correttive, nel periodo 2007-2012 è stata condotta dall'Osservatorio Nazionale per il Monitoraggio della Iodoprofilassi in Italia (OSNAMI) presso 1'Istituto Superiore di Sanità, una prima sorveglianza che aveva confermato il persistere di una lieve iodocarenza su gran parte del territorio nazionale [1]. In particolare, in quegli anni la vendita di sale iodato in Italia era inferiore al $50 \%$ di tutto il sale venduto, e nella popolazione in età scolare la concentrazione mediana di iodio urinario (Urinary Iodine Concentration, UIC) era risultata indicativa di iodosufficienza solo in tre (Liguria, Toscana, Sicilia) delle nove regioni esaminate (Piemonte, Lombardia, Veneto, Molise, Puglia, Calabria), mentre la prevalenza del gozzo era superiore al valore soglia del 5\% nelle 6 regioni

Proposto da A. Olivieri.

Extended author information available on the last page of the article che avevano fornito i dati (Liguria, Emilia-Romagna, Marche, Toscana, Calabria, Sicilia). Anche la frequenza di valori elevati di TSH neonatale aveva confermato una lieve carenza nutrizionale di iodio nella popolazione neonatale [1].

Sulla base di questi risultati, la Direzione Generale per l'Igiene e la Sicurezza degli Alimenti e la Nutrizione (DGISAN) del Ministero della Salute, insieme al panel di esperti dell'OSNAMI, hanno deciso di intensificare le campagne informative per la promozione del programma di iodoprofilassi attraverso la diffusione dello slogan POCO SALE MA IODATO, in accordo con la strategia di riduzione del consumo di sale, già attuata a livello nazionale dal Ministero della Salute. Tali sforzi, sostenuti anche dal continuo impegno nel promuovere il programma di iodoprofilassi da parte di Associazioni dei Pazienti, Società Scientifiche, medici dei Servizi per l'Igiene degli Alimenti e la Nutrizione (SIAN), hanno portato al raggiungimento della iodosufficienza, così come dimostrato dai risultati della seconda sorveglianza OSNAMI sintetizzati qui di seguito e riportati con dettaglio regionale in un recente report dell'Istituto Superiore di Sanità [2].

\section{Risultati della seconda sorveglianza OSNAMI (2015-2019)}

Nel periodo 2015-2019 l'OSNAMI, grazie alla collaborazione con gli Osservatori Regionali per la Prevenzione del Gozzo, ha condotto una seconda sorveglianza nazionale con l'obiettivo di valutare lo stato nutrizionale iodico della popolazione italiana. All'indagine hanno partecipato gli Osservatori di 10 regioni rappresentative del Nord (Liguria, Veneto, 
Tabella 1 Numerosità degli scolari italiani reclutati nello studio di sorveglianza stratificati per area di residenza (2015-2019)

\begin{tabular}{|c|c|c|c|c|}
\hline & $\begin{array}{l}\text { Nord } \\
\text { (Liguria, } \\
\text { Emilia-Romagna, Veneto) }\end{array}$ & $\begin{array}{l}\text { Centro } \\
\text { (Toscana, Marche, } \\
\text { Umbria, Lazio) }\end{array}$ & $\begin{array}{l}\text { Sud } \\
\text { (Molise, } \\
\text { Calabria, Sicilia) }\end{array}$ & Totale \\
\hline n. bambini reclutati & 1.407 & 1.615 & 1.211 & 4.233 \\
\hline Maschi (\%) & $738(52)$ & $815(50)$ & $662(55)$ & $2.215(52)$ \\
\hline Femmine $(\%)$ & $669(48)$ & $800(50)$ & $549(45)$ & $2.018(48)$ \\
\hline 11 anni $(\%)$ & $277(20)$ & $351(22)$ & $501(41)$ & $1.129(27)$ \\
\hline 12 anni $(\%)$ & $493(35)$ & $503(31)$ & $415(34)$ & $1.411(33)$ \\
\hline 13 anni $(\%)$ & $637(45)$ & $761(47)$ & $295(24)$ & $1.693(40)$ \\
\hline $\begin{array}{l}\text { n. bambini reclutati } \\
\text { in aree urbane }(\%)\end{array}$ & $426(30)$ & 919 (57) & $866(72)$ & $2.211(52)$ \\
\hline $\begin{array}{l}\text { n. bambini reclutati } \\
\text { in aree rurali }(\%)\end{array}$ & $981(70)$ & $696(43)$ & $345(28)$ & $2.022(48)$ \\
\hline
\end{tabular}

Emilia-Romagna), Centro (Toscana, Umbria, Marche, Lazio) e Sud del paese (Molise, Calabria, Sicilia). In 35 centri abitati distribuiti tra aree rurali $(\mathrm{n}=19)$ e aree urbane $(n=16)$ sono stati reclutati 4233 scolari di età compresa tra gli 11 e i 13 anni. Le numerosità dei bambini reclutati nelle diverse aree di residenza sono riportate nella Tabella 1.

\section{Valutazione dell'efficienza del programma di iodoprofilassi}

Il primo obiettivo dell'azione di monitoraggio è quello di valutare l'efficienza della iodoprofilassi, ovvero quanto iodio arriva alla popolazione. Tale obiettivo è raggiunto attraverso l'analisi dei dati di consumo di sale iodato e della concentrazione urinaria di iodio in età scolare.

\section{Percentuale di utilizzo del sale iodato}

Tutti i bambini reclutati per lo studio sono stati sottoposti a visita medica e ai genitori è stato somministrato un questionario per verificare l'uso di sale iodato. I dati hanno mostrato un progressivo aumento dell'utilizzo di sale iodato con una percentuale del $54 \%$ nel 2015 e del $71 \%$ nel quadriennio 2016-2019. Quest'ultimo dato è perfettamente sovrapponibile ai risultati ottenuti nello stesso quadriennio dal sistema di sorveglianza PASSI dell'Istituto Superiore di Sanità, che dal 2008 raccoglie informazioni sugli stili di vita e fattori di rischio comportamentali della popolazione adulta residente in Italia. Infatti, i dati derivanti dalle interviste condotte su oltre 130.000 soggetti adulti, hanno mostrato che il sale iodato veniva utilizzato dal 71,5\% degli intervistati [3].

Parallelamente ai dati di utilizzo del sale iodato da parte delle famiglie italiane, è importante rilevare il dato riguardante l'utilizzo di sale iodato nelle mense scolastiche (Fig. 1). Grazie al lavoro capillare di ispezione e promozione della iodoprofilassi svolto dai medici dei Servizi di Igiene degli Alimenti e della Nutrizione (SIAN) in questi anni, è risultato che il $78 \%$ delle 998 scuole ispezionate nel 2017 utilizzava sale iodato, con picchi del $97 \%$ in Sicilia e del $90 \%$ in Toscana, oltre a una percentuale del $94 \%$ a Bolzano dove la iodoprofilassi viene eseguita con successo dal 1982 [4]. Il dato sull'utilizzo del sale iodato nelle mense scolastiche è particolarmente importante se si pensa che per molti bambini il pasto principale è rappresentato proprio da quello che si consuma a scuola.

\section{Valori mediani di ioduria (UIC)}

La UIC è stata determinata mediante spettrometria di massa al plasma accoppiato induttivamente (Inductively Coupled Plasma-Mass Spectrometry, ICP-MS) su campioni estemporanei di urine (spot) raccolti in 3.510 bambini. Le determinazioni sono state effettuate dal Dipartimento di Medicina Clinica e Sperimentale, Sezione di Endocrinologia dell'Università di Pisa nei primi 2 anni di reclutamento, e dal Dipartimento di Sicurezza Alimentare, Nutrizione e Sanità Pubblica Veterinaria dell'Istituto Superiore di Sanità nel restante periodo di reclutamento. Al fine di confrontare i dati di ioduria ottenuti nella seconda sorveglianza mediante ICP-MS con quelli ottenuti con metodo spettrofotometrico (Sandell-Kolthoff) utilizzato nella prima sorveglianza, sono stati confrontati i due metodi. Il confronto ha mostrato un idoneo grado di accuratezza e riproducibilità delle misure ottenute con le due metodologie [5].

I risultati delle determinazioni della UIC hanno mostrato un valore mediano nazionale pari a $124 \mu \mathrm{g} / \mathrm{L}$ (range interquartile, IQR = 77-188) indicativo di uno stato di iodosufficienza. Inoltre, a livello nazionale non è stata evidenziata alcuna differenza tra i valori mediani di ioduria rilevati nelle aree rurali (UIC mediana $=129 \mu \mathrm{g} / \mathrm{L}, \mathrm{IQR}=82-196$ ) e in quelle urbane (UIC mediana $=119 \mu \mathrm{g} / \mathrm{L}, \mathrm{IQR}=72-180$ ), 
Fig. 1 Prevalenza di mense scolastiche in cui viene utilizzato il sale iodato (dati 2017)

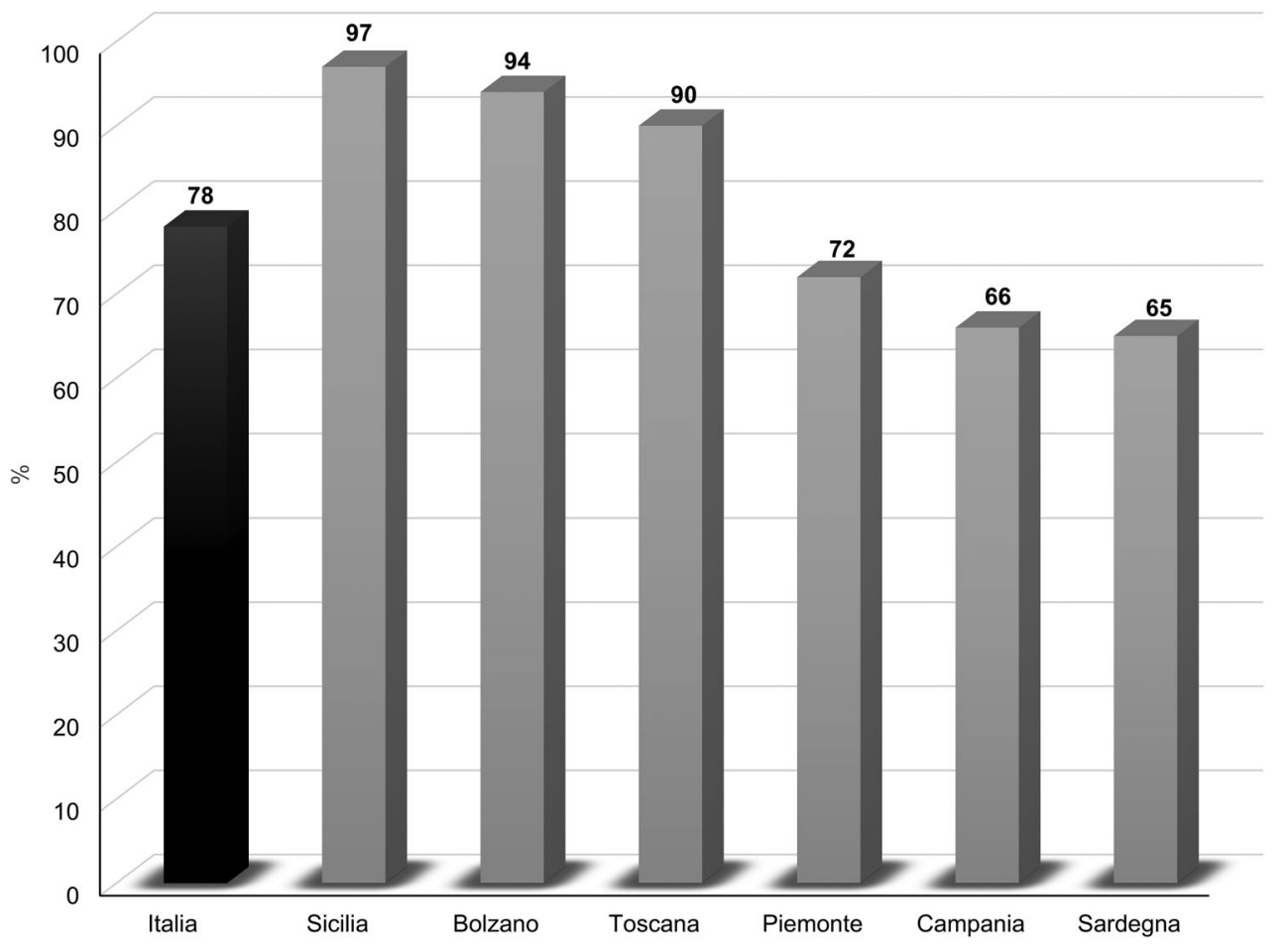

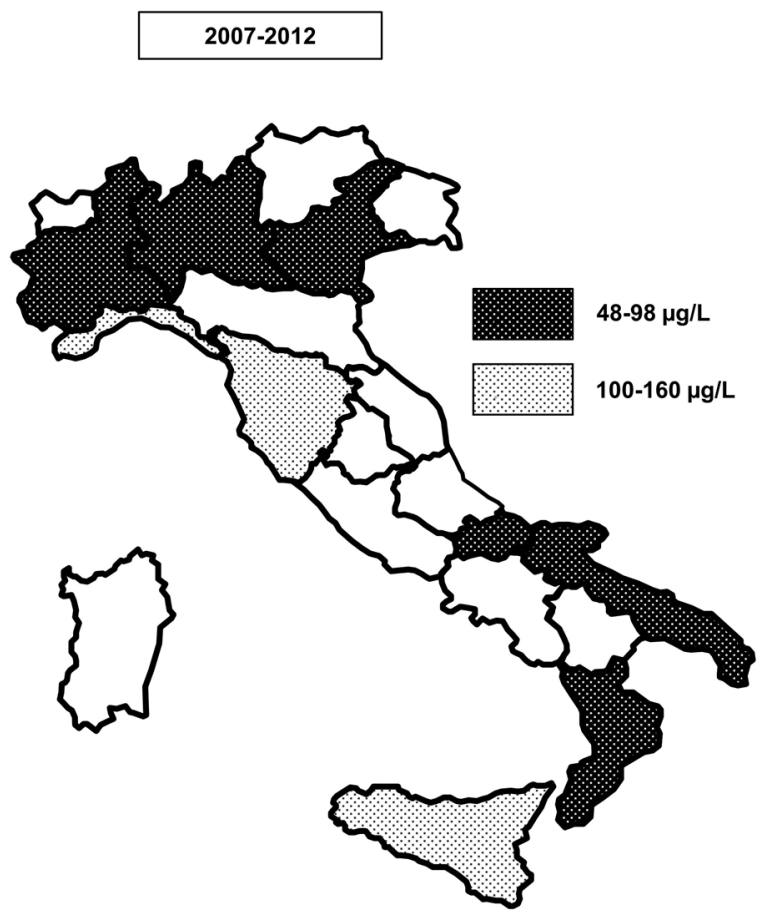



Fig. 2 Valori mediani di ioduria in bambini in età scolare (11-13 anni) rilevati durante la prima (periodo 2007-2012) e la seconda sorveglianza OSNAMI (periodo 2015-2019)

mentre a livello locale sono risultate lievemente iodocarenti solo l'area urbana della Sicilia $(89 \mu \mathrm{g} / \mathrm{L})$ e l'area rurale delle Marche ( $98 \mu \mathrm{g} / \mathrm{L})$, anche se è da sottolineare che questi ultimi dati fanno riferimento a un reclutamento avvenuto nel 2015 (Fig. 2).

\section{Dati sulla nutrizione iodica in gravidanza}

Durante il periodo 2015-2019 l'OSNAMI ha avviato lo studio multicentrico "Iodio-Gravidanza-Neonato" con la finalità di produrre dati epidemiologici di valenza nazionale re- 
lativi allo stato nutrizionale iodico in gravidanza e alla funzione tiroidea neonatale. Allo studio partecipano gli Osservatori Regionali di 8 regioni (Lombardia, Liguria, Veneto, Toscana, Lazio, Calabria, Sicilia e Sardegna) con un reclutamento previsto di 3.500 donne al momento del parto. I risultati preliminari di questo studio sono previsti per la fine del 2022.

Parallelamente, in 4 di queste regioni (Lombardia, Toscana, Sicilia, Sardegna) sono stati condotti studi preliminari che hanno fornito delle prime informazioni sulla percentuale di donne in gravidanza che fa uso di sale iodato e/o di integratori contenenti iodio. Su un totale di 1.984 donne gravide cui era stato somministrato uno specifico questionario, il $69,9 \%$ dichiarava di far uso di sale iodato e il $48,5 \%$ di usare integratori contenenti iodio da soli o in associazione al sale iodato. Questi dati sono coerenti con quelli ottenuti nello studio PASSI sopra citato, dal quale è risultato che il $75 \%$ delle donne in gravidanza e il $73 \%$ delle donne in allattamento intervistate faceva uso di sale iodato [3].

\section{Valutazione dell'efficacia del programma di iodoprofilassi}

Altro obiettivo del monitoraggio è la valutazione dell'efficacia della iodoprofilassi, ovvero la valutazione della riduzione della frequenza delle patologie connesse alla carenza nutrizionale di iodio nella popolazione.

\section{Frequenza di gozzo in età scolare}

Per la stima della prevalenza del gozzo è stata effettuata l'ecografia tiroidea in 3.430 bambini. Il volume totale della ghiandola è stato calcolato applicando la formula di Brunn $[6,7]$. Il gozzo è stato valutato per sesso e per età in accordo con i limiti di riferimento pubblicati dal WHO nel 1997 [8], ovvero gli stessi utilizzati durante la prima sorveglianza, al fine di rendere confrontabili i due set di dati.

La prevalenza di gozzo in età scolare è risultata dell' $1,66 \%$, senza alcuna differenza tra aree rurali $(1,61 \%)$ e aree urbane (1,70\%) (Fig. 3). Solo nell'area rurale dell'Umbria la prevalenza è risultata appena al di sopra del $5 \%$ (area rurale 5,4\%; area urbana 3,9\%), valore indicato dal WHO come soglia al di sopra della quale si parla di gozzo endemico. A tale proposito è importante ricordare che la prevalenza di gozzo è un indicatore di apporto di iodio a lungo termine perché, sebbene il volume tiroideo si riduca all'aumentare dell'assunzione di iodio, è necessario che $\mathrm{i}$ bambini siano nati e cresciuti in condizioni di iodosufficienza affinché la prevalenza di gozzo nella popolazione scolare possa raggiungere valori inferiori al 5,0\% [9]. Alla luce di tali considerazioni, la prevalenza di gozzo rilevata in Umbria è da considerarsi comunque un successo, in quanto la regione ha raggiunto la condizione di iodosufficienza solo di recente.

Per ciò che riguarda la prevalenza di noduli, questa è rimasta invariata tra la prima (range $1,0-3,0 \%$ ) e la seconda sorveglianza (range $1,0-3,5 \%$ ).

\section{Frequenza del TSH neonatale $>5,0 \mathrm{mUI} / \mathrm{L}$}

Altro indicatore utile per valutare l'efficienza della iodoprofilassi è il TSH neonatale [7]. Questo è indicativo di iodosufficienza nella popolazione neonatale e, indirettamente, nelle donne in gravidanza, quando la prevalenza di valori elevati $(>5,0 \mathrm{mUI} / \mathrm{L}$ ) non supera la soglia del $3 \%$ (con prelievo eseguito tra la terza e la quarta giornata di vita in neonati a termine). In Italia, il TSH neonatale viene determinato in tutti i neonati per lo screening neonatale dell'ipotiroidismo congenito. Pertanto, grazie alla collaborazione dei Centri di Screening di cinque regioni rappresentative del Nord, Centro e Sud del paese (Lombardia, Veneto-Verona, EmiliaRomagna, Marche, Calabria), l'OSNAMI ha analizzato i dati relativi al TSH di oltre 1.000.000 di nati in queste regioni tra il 2004 e il 2018 (Fig. 4). Anche in questo caso i risultati sono coerenti con il miglioramento della nutrizione iodica nella popolazione. Infatti, sebbene la prevalenza dei valori elevati di TSH ( $>5,0 \mathrm{mUI} / \mathrm{L})$ superi ancora la soglia del $3 \%$, il trend è in diminuzione (6,1\% nel 2010 vs 4,9\% nel 2018). Anche se incoraggianti, questi risultati comunque dimostrano che la gravidanza nel nostro paese ancora rappresenta una fase della vita ad alto rischio di carenza nutrizionale di iodio.

\section{Valutazione di eventuali effetti avversi}

Un altro importante obiettivo dell'azione di monitoraggio è quello di valutare eventuali effetti avversi del programma di iodoprofilassi, quali l'aumento dell'incidenza di ipertiroidismo e dell'autoimmunità tiroidea [10-12].

\section{Prescrizioni di farmaci anti-tiroidei}

Con la collaborazione dell'Osservatorio nazionale sull'impiego dei Medicinali (OsMed) presso l'Istituto Superiore di Sanità, è stato possibile analizzare la variazione percentuale delle prescrizioni di metimazolo in Italia tra il 2001 e il 2018, quale indicatore indiretto dei nuovi casi di ipertiroidismo. L'analisi dei dati a livello nazionale ha mostrato una riduzione delle prescrizioni del farmaco anti-tiroideo pari al 7,4\%. Seppure indirettamente, questo risultato suggerisce una progressiva riduzione dell'incidenza delle forme di ipertiroidismo essenzialmente dovuto ad autonomia nodulare, quale positiva e attesa conseguenza di una migliore nutrizione iodica nella popolazione [13]. 

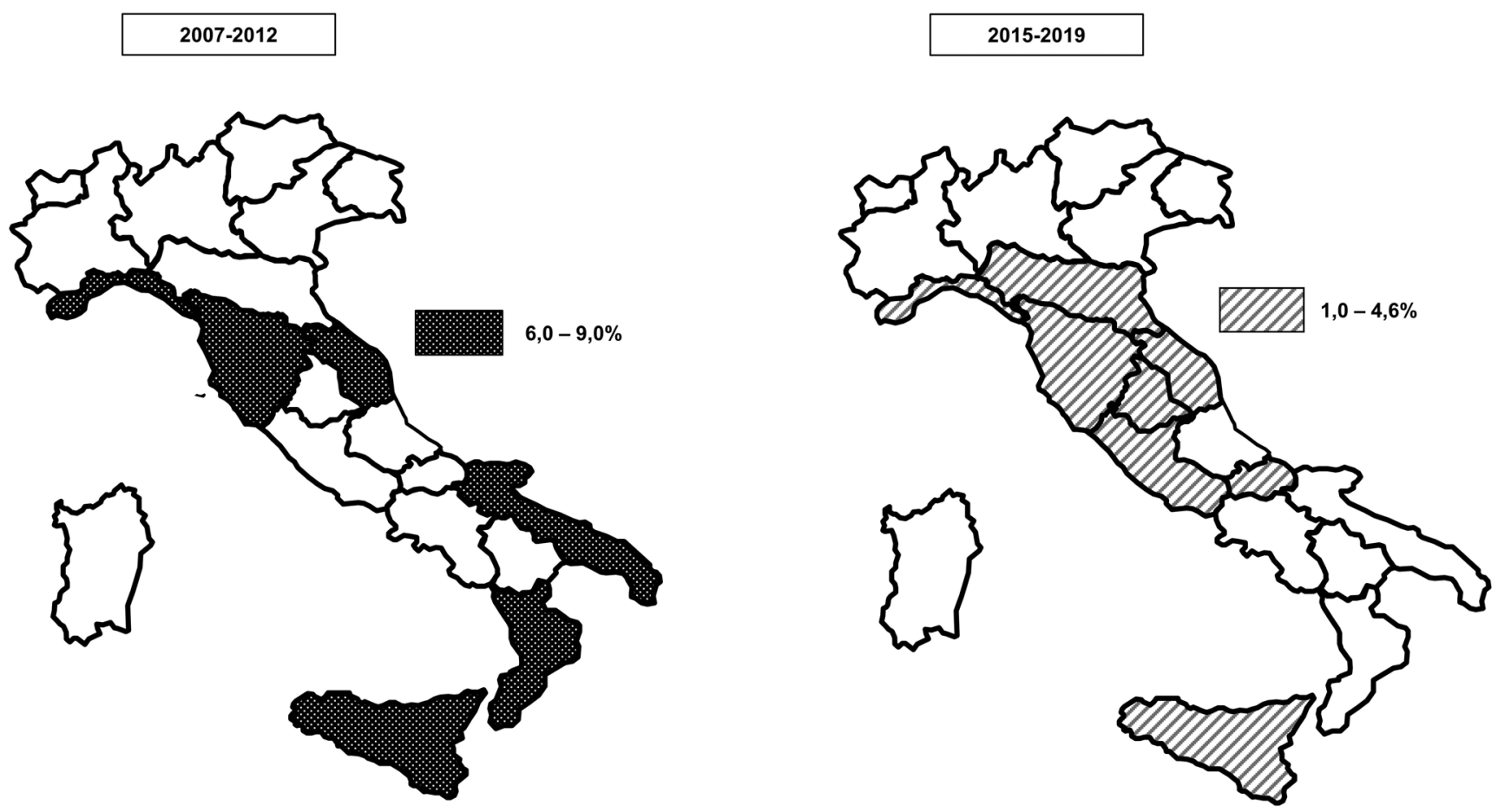

Fig. 3 Prevalenza di gozzo in bambini in età scolare (11-13 anni) rilevati durante la prima (periodo 2007-2012) e la seconda sorveglianza OSNAMI (periodo 2015-2019)

Fig. 4 Trend temporale della frequenza di TSH $>5,0 \mathrm{mUI} / \mathrm{L}$ in Italia
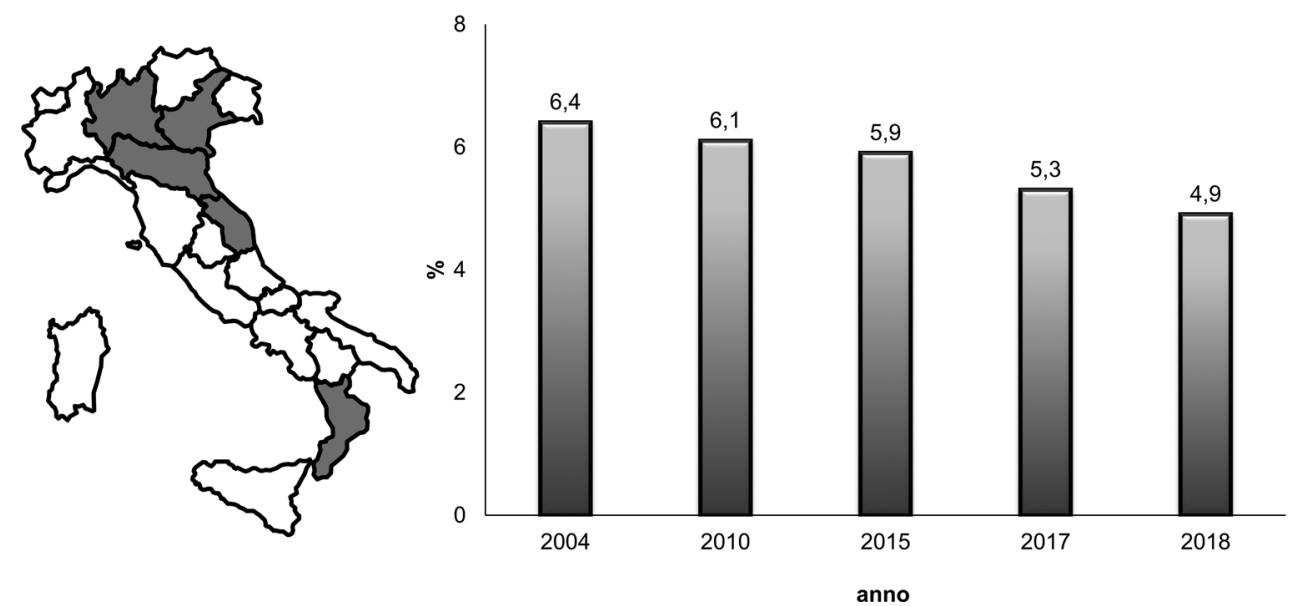

quindi, l'ecogenicità $[14,15]$. Pertanto, nei bambini residenti nelle tre regioni che avevano mostrato iodosufficienza già alla prima sorveglianza, ovvero Liguria, Toscana e Sicilia (area rurale), è stata anche valutata la frequenza di ipoecogenicità tiroidea. Sono state definite come ipoecogene quelle ghiandole rilevate come moderatamente o marcatamente ipoecogene.

L'analisi dei dati ha evidenziato una frequenza di ipoecogenicità del 6,6\% nelle tre regioni, senza differenze significative tra maschi $(7,8 \%)$ e femmine $(5,4 \%)$. Tuttavia, quando i dati sono stati stratificati per Body Mass Index (BMI) si è osservato che tale frequenza risultava significativamente $(p<0,001)$ inferiore nei bambini di peso adeguato $(4,4 \%)$ ria nel tessuto ghiandolare che ne modifica l'architettura e, 
rispetto ai bambini sovrappeso $(11,0 \%)$ e obesi $(24,1 \%)$, dimostrando un forte impatto del BMI su tale parametro. Questi risultati costituiscono una baseline della frequenza di ipoecogenicità in aree iodosufficienti da almeno 10 anni, che appare comunque contenuta nei soggetti normopeso, e suggeriscono l'importanza di valutare sempre il BMI come fattore confondente [16].

\section{Attività di promozione della iodoprofilassi}

Oltre all'attività di monitoraggio, ulteriore compito dell'OSNAMI è quello di promuovere la iodoprofilassi attraverso attività di formazione e informazione della popolazione.

\section{Position Statement sull'utilizzo del sale iodato in età adulta $\mathrm{e}$ in età pediatrica}

I risultati della prima sorveglianza, condotta nel periodo 2007-2012, avevano chiaramente mostrato che uno dei principali fattori che aveva rallentato il successo della iodoprofilassi era il persistere di ingiustificate resistenze nel raccomandare il sale iodato a tutta la popolazione. Per superare tale ostacolo, la DGISAN del Ministero della Salute e l'Istituto Superiore di Sanità attraverso l'OSNAMI hanno promosso la stesura di un documento di consenso sull'utilizzo del sale iodato in età adulta e in età pediatrica, quale efficace strumento di contrasto a condotte non favorenti il programma di iodoprofilassi. A tal fine, il 6 aprile 2017 è stato siglato da 14 tra Società Scientifiche e Associazioni un position statement in cui si esprime pieno consenso nel raccomandare a tutti l'uso di sale iodato, dal momento che ci sono evidenze sufficienti per ritenere che la quantità di iodio aggiunto al sale per uso alimentare nel nostro paese $(30 \mu \mathrm{g} / \mathrm{g})$ consente un apporto iodico adeguato anche in presenza di un consumo di sale contenuto nei limiti raccomandati dal WHO (5 g di sale negli adulti e meno nei bambini) [17].

\section{II progetto iodoprofilassi a scuola}

Dalla prima sorveglianza OSNAMI (2007-2012) era anche emerso che un ulteriore fattore che aveva rallentato il raggiungimento della piena efficienza del programma di iodoprofilassi era rappresentato dalla scarsa informazione di ampi strati della popolazione sull'importanza della iodoprofilassi. Pertanto, con lo scopo di incrementare l'informazione su questo importante tema di salute pubblica, per il triennio 2016-2019 è stato siglato un Protocollo di Intesa tra il Ministero dell'Istruzione, dell'Università e della Ricerca (MIUR), Istituto Superiore di Sanità, Associazione Italiana Tiroide (AIT), Associazione Medici Endocrinologi (AME), Società Italiana di Endocrinologia (SIE), Società Italiana di Endocrinologia e Diabetologia Pediatrica (SIEDP) e Comitato Associazioni Pazienti Endocrini (CAPE). Il Protocollo di Intesa ha previsto il progetto formativo "Progetto iodoprofilassi nelle scuole", rivolto a tutte le scuole primarie e secondarie di primo e secondo grado italiane. L'obiettivo era quello di consentire la formazione degli insegnanti sul tema della prevenzione dei disordini da carenza iodica, affinché formassero i loro studenti e questi, a loro volta, trasferissero le informazioni alle loro famiglie.

La realizzazione del progetto ha visto il coinvolgimento di oltre 28.000 studenti tra il 2017 e l'inizio del 2020, momento in cui il progetto è stato interrotto per l'insorgenza dell'emergenza sanitaria dovuta al Covid-19. Questo risultato conferma il successo dell'iniziativa che ha consentito di diffondere, presso le nuove generazioni e le loro famiglie, la cultura della prevenzione dei disordini da carenza iodica contribuendo al raggiungimento della iodosufficienza nel nostro paese.

\section{Sostegno alle campagne di informazione}

In questi anni l'azione di sostegno alle campagne di informazione presso la popolazione sull'uso di sale iodato è stata realizzata in accordo con la DGISAN del Ministero della Salute e con la cooperazione dei medici del territorio, i medici dei SIAN e il Comitato delle Associazioni Pazienti Endocrini (CAPE) che operano sul territorio nazionale, le quali contribuiscono in maniera determinante al processo di educazione della popolazione sull'importanza della iodoprofilassi. Inoltre, è stato costantemente aggiornato il sito web dell'OSNAMI (www.iss.it/osnami) i cui contenuti sono destinati non solo agli operatori del servizio sanitario nazionale, ma anche a tutti i cittadini che vogliano trovare informazioni semplici su carenza di iodio e iodoprofilassi nel nostro paese.

\section{Conclusioni}

I dati della seconda sorveglianza OSNAMI hanno documentato il raggiungimento della iodosufficienza nel nostro paese [18] e la scomparsa del gozzo in età scolare. È importante rilevare che tale risultato è stato raggiunto nonostante negli ultimi anni si sia osservata in Italia una riduzione del consumo di sale nella popolazione [19], confermando che la concentrazione di iodio nel sale commercializzato nel nostro paese $(30 \mathrm{mg} / \mathrm{kg})$ è, almeno per il momento, sufficiente a contrastare l'impatto di tale riduzione.

L'obiettivo che il paese ora dovrà porsi sarà quello di garantire la sostenibilità di questo importante programma di prevenzione. Ciò sarà possibile se si potrà realizzare un'incisiva azione di formazione che sia rivolta non solo alle nuove generazioni di medici, nutrizionisti e dietisti, ma anche agli studenti della scuola primaria e secondaria di primo e secondo grado, così come già sperimentato con successo durante questi anni. Allo stesso tempo sarà necessaria una più 
capillare e accurata azione di monitoraggio che preveda il coinvolgimento di tutte le regioni, di quei segmenti di popolazione a maggior rischio di iodocarenza, come le donne in gravidanza per le quali ad oggi mancano ancora dati epidemiologici di valenza nazionale, e un'accurata sorveglianza sul consumo di sale nella popolazione, al fine di valutare l'eventuale impatto che un'ulteriore riduzione del suo consumo potrebbe avere sull'efficienza della iodoprofilassi.

Informazioni Supplementari La versione online contiene materiale supplementare disponibile su https://doi.org/10.1007/s40619-02201018-3.

\section{Dichiarazioni etiche}

Conflitto di interesse Tutti gli autori dichiarano di non avere conflitti di interesse.

Consenso informato L'attività di monitoraggio della iodoprofilassi viene eseguita obbligatoriamente in base alla normativa vigente (Atto d'intesa Stato Regioni del 26/03/2009, GU Serie Generale n. 75 del $31 / 03 / 2009)$. Il reclutamento dei bambini oggetto dello studio è avvenuto a seguito della sottoscrizione del consenso informato da parte dei genitori.

Studi sugli animali Gli autori di questo articolo non hanno eseguito studi sugli animali.

\section{Bibliografia}

1. Olivieri A, Vitti P (2014) In: Attività di monitoraggio del programma nazionale per la prevenzione dei disordini da carenza iodica. Rapporti ISTSAN 14/6. Istituto Superiore di Sanità, Roma

2. Olivieri A, De Angelis S (2021) In: Monitoraggio della iodoprofilassi in Italia. Dati 2015-2019. Rapporti ISTISAN 21/6. Istituto Superiore di Sanità, Roma

3. Minardi V, Contoli B, Possenti V et al (2021) Consumo di sale iodato nella popolazione adulta italiana: il contributo del sistema di sorveglianza PASSI. In: Olivieri A, De Angelis S (eds) Monitoraggio della iodoprofilassi in Italia. Dati 2015-2019. Istituto Superiore di Sanità, Roma, pp 24-33

4. Franzellin F, Lucchin L (2017) La iodoprofilassi: l'esperienza della Provincia Autonoma di Bolzano (Alto Adige). Recenti Prog Med 108:90-97

5. Pastorelli A, De Angelis S, Bagnasco M et al (2018) Determinazione spettrofotometrica della concentrazione urinaria di iodio: valutazione di un circuito interlaboratorio e confronto con la spettrometria di massa al plasma. Biochim Clin 42(SS1):S87

6. Brunn J, Block U, Ruf G et al (1981) Volumetric analysis of thyroid lobes by realtime ultrasound. Dtsch Med Wochenschr 106:1338-1340
7. Organization (WHO), United Nations Children's Fund (UNICEF), International Council for Control of Iodine Deficiency Disorders (ICCIDD) (2007) Assessment of iodine deficiency disorders and monitoring their elimination: a guide for programme managers, 3rd edn. WHO, Geneva. http://whqlibdoc.who.int/publications/ 2007/9789241595827_eng.pdf. Accessed 14 September 2021

8. World Health Organization, International Council for Control of Iodine Deficiency Disorders (WHO \& ICCIDD) (1997) Recommended normative values for thyroid volume in children aged 6-15 years. Bull World Health Organ 75:95-97

9. Aghini-Lombardi F, Antonangeli L, Pinchera A et al (1997) Effect of iodized salt on thyroid volume of children living in an area previously characterized by moderate iodine deficiency. J Clin Endocrinol Matab 82:1136-1139

10. Burgi H, Kohler M, Morselli B (1998) Thyrotoxicosis incidence in Switzerland and benefit of improved iodine supply. Lancet 352:1034

11. Teti C, Panciroli M, Nazzari E et al (2021) Iodoprophylaxis and thyroid autoimmunity: an update. Immunol Res 69:129-138

12. Ruggeri RM, Trimarchi F (2021) Iodine nutrition optimization: are there risks for thyroid autoimmunity? J Endocrinol Invest 44(9):1827-1835

13. Aghini-Lombardi F, Fiore E, Tonacchera M et al (2013) The effect of voluntary iodine prophylaxis in a small rural community: the Pescopagano survey 15 years later. J Clin Endocrinol Metab 98:1031-1039

14. Rago T, Chiovato L, Grasso L et al (2001) Thyroid ultrasonography as a tool for detecting thyroid autoimmune diseases and predicting thyroid dysfunction in apparently healthy subjects. J Endocrinol Invest 24:763-769

15. Loy M, Cianchetti ME, Cardia F et al (2004) Correlation of computerized gray-scale sonographic findings with thyroid function and thyroid autoimmune activity in patients with Hashimoto's thyroiditis. J Clin Ultrasound 32:136-140

16. De Angelis S, Bagnasco M, Moleti M et al (2021) Obesity and monitoring iodine nutritional status in schoolchildren: is body mass index a factor to consider? Thyroid 31:829-840

17. Ministero della Salute, Istituto Superiore di Sanità (2017) Position Statement su "Uso di sale iodato in età adulta e in età pediatrica". Ministero della Salute, Roma. https://www.salute.gov.it/imgs/ C_17_pubblicazioni_2593_allegato.pdf. Accessed 14 September 2021. Accessed 14 September 2021

18. Olivieri A, Trimarchi F, Vitti P (2020) Global iodine nutrition 2020: Italy is an iodine sufficient country. J Endocrinol Invest 43(11): 1671-1672

19. Donfrancesco C, Lo Noce C, Russo O et al (2021) Trend of salt intake measured by 24-h urine collection in the Italian adult population between the 2008 and 2018 CUORE project surveys. Nutr Metab Cardiovasc Dis 31(3):802-813

Nota della casa editrice Springer Nature rimane neutrale in riguardo alle rivendicazioni giurisdizionali nelle mappe pubblicate e nelle affiliazioni istituzionali.

\title{
Authors and Affiliations
}

\author{
Simona De Angelis ${ }^{1}$. Daniela Rotondi ${ }^{1}$. Enzo Gilardi ${ }^{1}$. Paolo Stacchini ${ }^{2}$. Augusto Alberto Pastorelli ${ }^{2}$. \\ Angela Sorbo ${ }^{2}$. Marilena D'Amato ${ }^{2}$. Anna Chiara Turco ${ }^{2}$ - Emanuela Medda ${ }^{3}$. Roberto Da Cas ${ }^{4}$. Sebastiano Andò ${ }^{5}$. \\ Daniela Bonofiglio $^{5}$. Marcello Bagnasco ${ }^{6}$. Maurizio Gasperi ${ }^{7}$. Domenico Meringolo ${ }^{8}$ - Caterina Mian ${ }^{9}$. \\ Efisio Puxeddu ${ }^{10}$. Concetto Regalbuto ${ }^{11}$. Mariacarla Moleti ${ }^{12}$. Augusto Taccaliti ${ }^{13}$. Salvatore Ulisse ${ }^{14}$.
}


Massimo Tonacchera ${ }^{15}$ - Maria Laura Tanda ${ }^{16}$. Francesco Boi ${ }^{17}$ • Valeria Ruggiero ${ }^{17}$. Stefano Mariotti ${ }^{17}$. Carlo Corbetta $^{18}$. Renzo Ciatti ${ }^{19}$. Elisabetta Tarsi ${ }^{19}$. Vera Stoppioni ${ }^{19}$. Nicola Perrotti ${ }^{20}$. Onorina Marasco ${ }^{21}$. Giovanna Scozzafava ${ }^{21}$. Marta Camilot ${ }^{22}$. Francesca Teofoli ${ }^{22}$. Francesca Righetti ${ }^{23}$. Antonio Dimida15 . Giuseppe Plutino $^{24}$. Elena Carrano ${ }^{24}$. Roberto Copparoni ${ }^{24}$ - Marco Gabbianelli ${ }^{1}$. Paolo Vitti ${ }^{15}$. Antonella Olivieri ${ }^{1}$

$凶$ A. Olivieri

antonella.olivieri@iss.it

1 Dipartimento di Malattie Cardiovascolari,

Endocrino-Metaboliche e Invecchiamento, Istituto Superiore di Sanità, Roma, Italia

2 Dipartimento di Sanità Pubblica Veterinaria e Sicurezza Alimentare, Istituto Superiore di Sanità, Roma, Italia

3 Centro di Riferimento per le Scienze Comportamentali e la Salute Mentale, Istituto Superiore di Sanità, Roma, Italia

4 Centro Nazionale Ricerca e Valutazione Preclinica e Clinica dei Farmaci, Istituto Superiore di Sanità, Roma, Italia

5 Centro Sanitario, Dipartimento di Farmacia e Scienze della Salute e della Nutrizione, Università della Calabria, Cosenza, Italia

6 Dipartimento di Medicina Interna e Specialità Mediche, Università di Genova, IRCCS Ospedale Policlinico S. Martino, Genova, Italia

7 Dipartimento di Medicina e Scienze per la Salute, Università degli Studi del Molise, Campobasso, Italia

8 Unità di Endocrinologia, Istituto Ramazzini, Bologna, Italia

9 Dipartimento di Medicina, Azienda Ospedaliera di Padova, Padova, Italia

10 Dipartimento di Medicina, Università degli Studi di Perugia, Perugia, Italia

11 Dipartimento di Biomedicina Clinica e Molecolare, Università di Catania, Catania, Italia

12 Dipartimento di Medicina Clinica e Sperimentale, Università degli Studi di Messina, Messina, Italia
13 Clinica di Endocrinologia e Malattie del Metabolismo, Università Politecnica delle Marche, Ancona, Italia

14 Dipartimento di Scienze Chirurgiche, "Sapienza" Università di Roma, Roma, Italia

15 Dipartimento di Medicina Clinica e Sperimentale, Università di Pisa, Pisa, Italia

16 Dipartimento di Medicina e Chirurgia, Università degli Studi dell'Insubria, ASST-Settelaghi, Varese, Italia

17 Dipartimento di Scienze Mediche e Sanità Pubblica, Università degli Studi di Cagliari, Cagliari, Italia

18 Laboratorio di Riferimento Regionale per lo Screening Neonatale, Ospedale V. Buzzi, Milano, Italia

19 Centro Screening Neonatale Regione Marche, UOC di Neuropsichiatria Infantile, Azienda Ospedaliera Ospedali Riuniti Marche Nord, Presidio Ospedaliero Santa Croce, Fano, Italia

20 Dipartimento Scienze della Salute, Università Magna Graecia, Catanzaro, Italia

21 Laboratorio Regionale di Screening Neonatale, Azienda Ospedaliera Mater Domini, Catanzaro, Italia

22 Laboratorio Regionale di Screening Neonatale, Azienda Ospedaliera Universitaria Integrata di Verona, Ospedale Borgo Roma, Verona, Italia

23 Centro Laboratoristico Regionale di Riferimento per lo Screening Neonatale e Malattie Endocrino-Metaboliche, Azienda Ospedaliero-Universitaria Policlinico Sant'Orsola-Malpighi, Bologna, Italia

Direzione Generale per l'Igiene e la Sicurezza degli Alimenti e la Nutrizione, Ministero della Salute, Roma, Italia 